\title{
BMJ Open Refused and referred-persistent stigma and discrimination against people living with HIV/AIDS in Bihar: a qualitative study from India
}

\author{
Mohit Nair, ${ }^{1}$ Pragya Kumar, ${ }^{2}$ Sanjay Pandey, ${ }^{2}$ Amit Harshana, ${ }^{1}$ Shahwar Kazmi, ${ }^{1}$ \\ Laura Moreto-Planas, ${ }^{1}$ Sakib Burza (1) ${ }^{1}$
}

To cite: Nair M, Kumar $P$, Pandey S, et al. Refused and referred-persistent stigma and discrimination against people living with HIV/ AIDS in Bihar: a qualitative study from India. BMJ Open 2019;9:e033790. doi:10.1136/ bmjopen-2019-033790

- Prepublication history for this paper is available online. To view these files, please visit the journal online (http://dx.doi. org/10.1136/bmjopen-2019033790).

Received 22 August 2019 Revised 30 0ctober 2019 Accepted 07 November 2019

Check for updates

(c) Author(s) (or their employer(s)) 2019. Re-use permitted under CC BY-NC. No commercial re-use. See rights and permissions. Published by BMJ.

${ }^{1}$ Medecins Sans Frontieres, New Delhi, India

${ }^{2}$ Community \& Family

Medicines, All India Institute of Medical Science, Patna, Bihar, India

Correspondence to

Dr Sakib Burza;

Sakib.Burza@barcelona.msf.org

\section{ABSTRACT}

Objectives This study aimed to explore barriers to accessing care, if any, among people living with HIV/AIDS (PLHA) in two districts of Bihar. We also aimed to assess attitudes towards PLHA among healthcare providers and community members.

Design This qualitative study used an exploratory study design through thematic analysis of semistructured, indepth interviews.

Setting Two districts were purposively selected for the study, namely the capital Patna and a peripheral district located approximately $100 \mathrm{~km}$ from Patna, in order to glean insights from a diverse sample of respondents.

Participants Our team purposively selected 71 participants, including 35 PLHA, 10 community members and 26 healthcare providers.

Results The overarching theme that evolved from these data through thematic coding identified that enacted stigma and discrimination interfere with each step in the HIV care continuum for PLHA in Bihar, India, especially outside urban areas. The five themes that contributed to these results include: perception of HIV as a dirty illness at the community level; non-consensual disclosure of HIV status; reliance on identifying PLHA to guide procedures and resistance to universal precautions; refusal to treat identified PLHA and referrals to other health centres for treatment; and inadequate knowledge and fear among health providers with respect to HIV transmission.

Conclusions The continued presence of discriminatory and stigmatising attitudes towards PLHA negatively impacts both disclosure of HIV status as well as access to care and treatment. We recognise a pressing need to improve the knowledge of HIV transmission, and implement universal precautions across all health facilities in the state, not just to reduce stigma and discrimination but also to ensure proper infection control. In order to improve treatment adherence and encourage optimal utilisation of services, it is imperative that the health system invest more in stigma reduction in Bihar and move beyond more ineffective, punitive approaches.

\section{INTRODUCTION}

Stigma and discrimination associated with HIV/AIDS are conceptualised as processes of devaluation of people either living with
Strengths and limitations of this study

- To our knowledge, this is the first study from this region of India to document refusals and referrals of people living with HIV/AIDS (PLHA) in the healthcare system, despite legal protections for PLHA under the new law.

- This is one of the first studies to document that universal precautions are widely misunderstood in this region and PLHA are routinely asked to purchase infection control supplies prior to surgery.

- The study includes an urban city centre as well as a more remote district $100 \mathrm{~km}$ away to purposively sample for heterogeneity. However, the sample is limited to two districts.

- We were unable to assess intersecting stigmas for key populations, such as transgender individuals, men who have sex with men, or injecting drug users, as participants in the study. These groups were much harder to target and our study design was limited to patients and community members presenting to healthcare facilities.

- Sample selection may present a source of bias, and caution must be exercised in generalising our findings. We relied on a subjective interpretation of self-reported data and were unable to independently verify any of the claims made by participants, but we mitigated this through triangulation by interviewing a multitude of stakeholders within the healthcare system in Bihar.

or associated with HIV and AIDS. ${ }^{1}$ Globally, key populations and high-risk groups, such as transgender women, migrant workers, men who have sex with men (MSM) or sex workers, face particularly high levels of stigma and discrimination. ${ }^{23}$

Most research studies that examine stigma typically fall into one of three broad categories: (1) enacted stigma, where people experience and report stigma first hand, ${ }^{45}$ (2) anticipated stigma, where people anticipate stigma on disclosure of HIV status ${ }^{67}$ and 
(3) internalised stigma, where people absorb and start to believe negative attitudes about themselves. ${ }^{8}{ }^{9}$ Experiences with enacted stigma and its associations with treatment delays and poor mental health outcomes are reported in multiple studies conducted across Southern India and Maharashtra. ${ }^{45-17}$ Ekstrand et al found that $89 \%$ of doctors, $88 \%$ of nurses and $73 \%$ of ward staff reported that they would discriminate against people living with HIV/AIDS (PLHA) in situations with a high likelihood of fluid exposure. ${ }^{15}$ The vast majority of studies are from Southern India, but very few focus on North India: evidence from Bihar, a populous state of 110 million, is very limited. This is a particular gap considering that Bihar has a large share of socioeconomically disadvantaged and economic migrants.

Stigma reduction strategies at the policy level have largely focused on punitive measures. For example, the Indian Government recently passed the HIV and Acquired Immune Deficiency Syndrome (Prevention and Control) Act in 2017, which made it illegal to discriminate against PLHA. ${ }^{18}$ According to the provisions of the Act, violations such as denial of medical services will result in imprisonment ranging from 3 months to 2 years or a maximum fine of Rs100 000 (approximately US\$1400) or both. ${ }^{18}$

The HIV epidemic has been largely concentrated in high-risk populations, but the large migrant population in Bihar poses significant risks to containing the spread of HIV. The HIV Estimation 2017 Report indicates that AIDS-related mortality has been steadily rising in Bihar; Bihar is also 1 of 10 states that account for $71 \%$ of total annual new HIV infections. ${ }^{19}$ In such a vulnerable context, it is important to understand the perceptions of HIV and access to care from both end user and provider perspectives in order to mitigate any barriers and work towards a more patient-centred system of care and management. The present study bridges this gap by examining barriers to accessing care and treatment among PLHA in Bihar, including specific experiences of stigma, and triangulating it with attitudes towards PLHA among healthcare providers and community members.

\section{METHODS}

\section{Study locations}

Two districts were purposively selected for the study, namely the state capital Patna and another predominantly rural and peripheral district located within $100 \mathrm{~km}$ of Patna, in order to glean insights from a diverse sample of respondents (in terms of age, sex and geographical location). The peripheral district caters to a significant migrant population, which makes it a pertinent choice for the study as the HIV epidemic in India is concentrated predominantly in particular high-risk, bridge populations (including migrants). Considering the limited number of health facilities in the rural district, we have chosen to withhold its name in order to protect healthcare providers' identities. We also chose to include Patna, given that it has several high-profile tertiary care and treatment centres. Furthermore, the capital also attracts people from all corners of the state who do not wish to seek care in close proximity to their homes, out of concerns for privacy regarding their HIV status, through forced referral or through perceptions of better quality care, making it a priority area for maximising representation in our study.

\section{Study design}

This qualitative study used an exploratory study design to uncover barriers to accessing care. We conducted semistructured, in-depth interviews with PLHA, healthcare personnel, as well as community members accessing care at health facilities across two districts of Bihar in order to capture a diverse study population and triangulate study findings.

First, PLHA were purposively sampled for participation in the study on the following basis:

- >18 years of age.

- Able and willing to provide informed consent.

- Living with HIV.

They were approached and recruited from the government and private health facilities providing outpatient department (OPD) or inpatient department services. Participants were approached while they were waiting to seek services, informed about the study and asked whether they would be interested in participating. All interviews took place in a private area of the health facility, away from other patients. Written informed consent was obtained from each patient, and all participants were reimbursed for the loss of daily wages (calculated as an average of Rs300 per participant).

Second, in-depth interviews were conducted with health staff who provide some level of care to PLHA, which included doctors, nurses and counsellors from both the public and private centres. There were no other specific eligibility criteria for these participants. Two researchers recruited these participants through snowball sampling directly from the health facilities. The first set of providers was recruited based on a list of providers compiled by the researchers. Subsequent providers were recruited through snowball sampling, with a special emphasis on obtaining a diverse array of medical specialties, gender and experience. All respondents were interviewed on a one-to-one basis, but we conducted one group interview with three healthcare providers at their private clinic as they expressed they did not have time to be interviewed separately.

Finally, the study aimed to gauge the attitudes of community members towards PLHA. As a result, community members who were over the age of 18 and accessing general OPD services were also recruited for in-depth interviews. All community members above the age of 18 were eligible for participation, provided they (1) did not identify as a PLHA (self-reported) and (2) did not have a family member with HIV (self-reported). The rationale behind this selection criteria was to try and obtain 
insights from a group of participants who may not otherwise interact with PLHA on a routine basis.

\section{Patient and public involvement}

Patients or the public were not involved in the design, or conduct, or reporting of the research, but preliminary results were disseminated to the public in workshops and conferences.

\section{Data collection and analysis}

The semistructured interview guide explored broad domains of care and treatment, including behaviour and attitudes towards patients, but the interview guide was flexible in nature in order to allow for newer areas of inquiry to be inductively derived from the data. All interviews were audio recorded with the written consent of the interviewee with the exception of two respondents who did not wish to be audio recorded. In this case, the interviewers took extensive notes during the interview process. Patients were continuously sampled until theoretical saturation (ie, the point at which no new insights emerge from the data) was attained. Since data collection and analysis occurred concurrently, we were able to assess how and when themes were saturated and we stopped conducting additional interviews when no new information was being reported by the participants.

All audio recordings were transcribed and translated (from Hindi, Bhojpuri, Magahi, Maithili or associated languages) into English, before being entered into NVivo qualitative data analysis software for in-depth coding and analysis. Sections of the transcripts were back-translated at random by an independent, multilingual researcher to assess transcription quality. Following data transcription, translation and entry into NVivo software, two researchers who are well trained in qualitative methods reread transcripts several times. None of the respondents had interacted with any of the participants before. As categories and themes started to emerge, two researchers engaged in an open and axial coding process as part of an inductive, thematic analysis: codes and categories pertaining to perceptions of care and treatment were inductively derived from the data and were followed by development of subcategories. Axial coding enabled us to regroup data in different ways and examine patterns and relationships between codes. The final coding framework was iteratively developed and broader categories informed the development of a broader theme with respect to the role of stigma and discrimination.

\section{RESULTS}

In total, 71 participants were interviewed, including 35 PLHA, 10 community members and 26 healthcare providers. Fifty-one respondents were male, and 20 were female, with respondents ranging in age from 21 to 66 years. Among medical staff, many different levels of seniority and medical specialisations were represented, including medical officers, pathologists, anaesthetists, radiologists, obstetrician-gynaecologists and surgeons, among others.

The interviews yielded rich narratives, which were inductively coded, categorised and subsequently classified into five key thematic areas grounded in the data. The results section elucidates each of the five themes in turn and provides narrative examples triangulated between stakeholders as justification.

\section{Enacted stigma at the community level: society conceptualises HIV as a 'dirty' or 'immoral' illness}

Community members had very limited knowledge about HIV and modes of transmission, but the vast majority associated HIV with 'immoral' or 'dirty' acts that reflected the poor character of PLHA: 'Society sees them [PLHA] with disgust. That he is not a good person, or he is a morally incorrect man and does not behave well. They see the family from a bad lens. Women are viewed even more negatively in comparison to men: they are seen as being improper' [CM9, Male, Age 22]. 'There is nobody I personally know with HIV in the village, but people who are uneducated or don't know much often look at PLHA with disgust or discriminate against them...some people think people living with HIV are 'chu-a-choot' (untouchable). They ask other people with HIV to eat separately and isolate them, or drink chai from a different cup' [CM3, Male, Age 40].

Misconceptions about the modes of transmission related to HIV were very common. Many community members reported that food and drinks should not be shared with PLHA: 'you get AIDS from sharing food as well...they should stay a little far away' [CM6, Male, Age 24]. Others mentioned that they would not buy vegetables from PLHA, or eat food prepared by PLHA. While some people understood that HIV is spread through direct contact with blood, many generalised this fear and expressed that they would have to 'stay at least a little far away from PLHA. Even if it is a close friend, I will have to stay a little far away' [CM4, Male, Age 25]. It was commonly perceived by community members that HIV was a dangerous, life-threatening illness with no cure: 'the only thing I have heard about HIV/AIDS, madam, is that...like, [it involves] wrong injections, wrong relations between people...it is a life-threatening illness due to these behaviours. It is a life threatening illness coming from these factors' [CM1, Male, Age 38].

Finally, PLHA also commonly reported feeling like they were considered untouchable as a result of HIV infection: 'people hate us. They will not sit with us, won't eat together, and will hate us and think that they will also contract the disease" [Patient 24, Male, Age 32]. Some respondents internalised such stigma and refrained from disclosing details about their illness to others due to anticipated stigma: 'I feel as if it's my mistake. How did this illness come inside me? ... I don't tell people because if they find out, they will feel disgust towards me...[thinking] he must have committed some wrong deed, which is why he has this illness' [Patient 17, Male, Age 35]. Hence, 
experiences of enacted stigma shaped the ways in which participants internalised stigma and self-isolated themselves from family members: 'The glass in which I drink water-I throw the remaining water. I don't allow anyone to drink that, or eat from the plate in which I eat food. If food is left, it is thrown away...my leftovers are not given to anyone...I worry about other people getting the same disease' [Patient 3, Male, no age reported].

These responses were also triangulated through interviews with healthcare providers: 'The social aspect is a very big factor, where the patient himself is very scared to explain to other people that he is HIV positive. He keeps this hidden. And then people also think that suppose I touch someone who is HIV positive or I'm exposed, there is this fear that I will also have trouble explaining how I got HIV to other people' [HCP 24, Anaesthetist, Male, no age reported]. PLHA anticipated stigmatising encounters in healthcare and community-based settings and often limited open disclosure of seropositive status: 'I have only told my family, since society thinks of this disease as filth' [Patient 1, Male, Age 44].

\section{Intentional and non-consensual disclosure of HIV status}

Despite PLHA wishing to keep their HIV status hidden from others, more than half of all doctors interviewed routinely informed the relative accompanying the patient of the HIV diagnosis before informing the patient: 'What we try to do is that...we do not tell the patient [about the HIV test result]. We tell the attendant [relative]. There is a psychological thing if you tell the patient. If I am alright right now, and someone tells me right now that I am about to have a heart attack, naturally I will get scared. So, first we do not inform the patient. We tell the attendant only. And then the attendants convey [to the patient]' [HCP 23, Radiologist, Male, Age 65]. All doctors who were interviewed considered themselves to be acting in the best interest of the patient in order to protect the patient from the shock of an HIV diagnosis. However, patients routinely claimed that these tests were conducted without their consent. For example, one patient, when asked if they were told what they were being tested for, claimed: 'no, I was not asked anything like this' [Patient 35, Male, Age 38]. He also stated that the test results were explained to his brother instead of him: 'they told my brother. I found out 2-4 days later that this illness has caught me.' Other patients reported similar narratives: 'nephew and brother were told that the test was satisfactory...they told to my family, not me' [Patient 27, Male, Age 30]. PLHA routinely experienced disclosure without their informed consent, which not only made them uncomfortable, but also led to worries of anticipated stigma in future encounters given past experiences of enacted stigma, as detailed in the previous thematic. 'They hate people with this disease. That much I have understood, Sir...they gave us less time compared to other patients...in [remote district name redacted], they used to hate HIV patients, not so much here [in Patna]...they [nurses] say you have come with a bad disease" [Patient 27, Male, Age 30].
Mandatory testing for HIV rather than universal precautions

Since patients were conceived to intentionally withhold information about their HIV status from doctors, routine and mandatory testing prior to admitting patients for surgery was commonly reported. Rather than following universal precautions, doctors tested all surgical patients for HIV and then adopted extra precautionary measures accordingly. The lack of gloves and other protective equipment at government facilities exacerbated this practice, and meant that doctors often requested patients to purchase gloves at their own expense. In the private healthcare system, doctors reported billing patients for the additional cost of protective equipment, such as gloves. This phenomenon was reported in the capital city as well as remote corners of the district:

See, it is an unsaid rule, okay? Patients will not be taken for surgery-there are unsaid rules-you get every patient screened for HIV [HCP 13, Orthopaedic surgeon, Male, Age 40]. I screen and conduct viral marker tests for each and every patient before taking them in the operation theatre [HCP 14, General surgeon, Male, Age 38].

Private sector physicians operating their own clinics usually asked patients to purchase their own separate disposable kits-it was stated that a single operation can typically be managed with 2-3 surgical kits. 'Like, in one kit, there are two gowns-one for the surgeon and one for the assistant. And then, [you need it] for the anaesthetist also, so we need two kits. One kit comes for around 300-400 rupees...less than 400 rupees' [HCP 24, Anaesthetist, Male, no age reported]. Additionally, since some paramedical staff are very hesitant to perform surgeries on PLHA, they receive extra incentives in the private sector: 'They have that somewhere in their minds, so we pay them a little bit extra. So, if a normal procedure is costing Rs20 000, that cost increases to Rs25 000-Rs27 000 . Meaning, including surgeon and anaesthetist and all extra charges, it will (cost) at least 5000-10 000 rupees extra, taking everything together' [HCP 24, Anaesthetist, Male, no age reported]. Patients confirmed that tests were usually undertaken before any operations, but they often could not report the purpose of these tests: "we had a blood test before the operation...I was told that HIV testing will be done...blood was taken for the test, and after two days, the operation was done' [Patient 27, Male, Age 30]. Mandatory testing also linked to subsequent refusals to treat PLHA: 'First, he tested the whole body...but I was not told anything. After saying nothing, he asked me where I used to live. I said Kolkata. Then, he prescribed two tests: HIV-1 and HIV-2. He asked me what I do...he tested me for a third time and then he rushed me out the door' [Patient 28, Male, Age 34]. This phenomenon is explored in greater detail as an independent thematic below. 
PLHA are often refused treatment and referred to higher centres

One of the most consistent themes emerging from patients and healthcare providers alike was that PLHA were usually referred onwards to tertiary centres rather than being treated at the particular facility where they presented, regardless of the severity of the condition. This held true across multiple specialisations of healthcare providers, but was more commonly seen outside the capital city of Patna. For instance, multiple healthcare providers confirmed that no surgical cases were admitted at one particular district hospital within $100 \mathrm{~km}$ of Patna, and that all such patients were referred to Patna for treatment: 'we make up some other excuse [to deny the patient]. Like we make an excuse saying I don't have any implants. I don't have that facility over here. That is why I will not be able to do this operation. I don't have that much knowledge. All these things we say' [HCP 26, Orthopaedic surgeon, Male, Age 44]. A senior medical officer at the same district hospital confirmed: 'we don't take any admission of HIV patients. They are sent to Patna' [HCP 2, Senior medical officer, Male, Age 60]. Even tertiary centres occasionally refer cases onward to missionary hospitals in order to avoid responsibility.

The situation in the peripheral or remote districts was generally perceived to be worse, largely due to the absence of resources. 'I refer them to a higher centre because on the level in which we are practicing, there is no such facility to handle HIV positive patients [PLHA]. For them, a separate kit is needed, everything is required separately, and we are not having that...so if I come through any such type of HIV positive patient [PLHA], or I suspect anyone even $1 \%$ also, then I refer them to a higher center' [HCP 17, Dentist, Male, Age 37]. Patients interviewed in the peripheral district reported that they were often referred for treatment: 'this doctor in [name redacted] where I was being treated for fever...he only wrote that I should go to Patna' [Patient 6, Male, Age 38]. Travelling for medication and treatment often took a toll on socioeconomically disadvantaged households: 'there were many problems in travelling... there were also problems in getting food. I am not from a big family, so there were many financial problems' [Patient 23, Male, Age 27].

The situation in the private sector was perceived to be worse than the government setup based on patient interviews. In the private sector, patients report feeling like they are perceived as being untouchable: 'since this is the illness, you know that doctors see you as untouchable... they don't even let us enter the room-let alone letting us sit down...he [doctor] says this is a chu-a-choot bimari [an illness of untouchability]...even now if I go anywhere in the private setup, and if I say upfront that I am an ART patient, they become hyper vigilant. You will not get to sit down on the seat- they will make you stand as they prescribe medicine" [Patient 26, Male, Age 55].
Inadequate knowledge of HIV transmission among health providers contributes to fear and stigmatising behaviours

One of the major drivers of stigmatising behaviours is a pervasive fear of HIV among medical professionals, including ward attendants, nurses, junior doctors and senior doctors, regarding occupational exposure to HIV. One private sector anaesthetist described this fear for us during a group interview with an obstetrician-gynaecologist and orthopaedic surgeon at their private clinic:

"Have you seen an advanced HIV patient? The way his body wastes away, his gaunt appearance, his papules everywhere...it is a truly scary sight. Have you worked with them? When you see a patient like this, you are truly scared" [HCP 24, Anaesthetist, Male, no age reported].

'There is no permanent cure for HIV. What can be done if $\mathrm{CD} 4$ count is reduced? And the taboo of AIDS in society is very scary. So, HIV is more dangerous" [HCP 15, Senior medical officer, Male, Age 47]. This often translates into direct refusals as one obstetrician demonstrated: "I have not taken HIV positive cases. I should not lie. I have not taken HIV cases out here" [HCP 25, OBGYN, Female, no age reported]. Yet another doctor reported that HIV cases were different because he feared how he would be perceived if he accidentally contracted HIV due to a needle prick: 'If I become HIV positive, more than $95 \%$ of people will think that I got it through sexual transmission...only technical people from the medical field will understand that it happened because I do many surgeries. But the majority of people will correlate it with your character' [HCP 10, General surgeon, Male, Age 35]. Another surgeon revealed that her husband and in-laws subjected her to HIV testing prior to marriage and continued to insist on regular testing at periodic intervals, since she chose to operate on PLHA. In this manner, the lack of a cure for HIV and the social stigma associating HIV with poor moral character compound the fears of doctors when it comes to operating on PLHA.

Taken together, these semistructured interviews help to inform an overarching theme: enacted stigma and discrimination inhibit access to healthcare for PLHA in Bihar, particularly outside urban areas. On the one hand, HIV is conceptualised as a dirty illness at the community level, while on the other, inadequate knowledge of HIV transmission and poor adoption of universal precautions among healthcare providers contribute to stigmatising behaviours towards PLHA at healthcare facilities. This often leads to treatment denial for PLHA and unnecessary onward referrals to tertiary facilities. This is especially salient among private healthcare providers in peripheral areas outside the capital city of Patna, even though tertiary care facilities in Patna are also far from accepting of PLHA.

\section{DISCUSSION}

In the process of inquiring about barriers to accessing care and treatment, this study uncovered both covert and overt forms of stigma and discrimination in the healthcare 
system. We found that enacted stigma was commonly reported by PLHA and discrimination at the health facility level inhibited access to healthcare for PLHA in Bihar by resulting in implicit denials of treatment and unnecessary referrals to tertiary care facilities, particularly outside urban areas. This was reportedly driven by inadequate knowledge of the modes of HIV transmission and a concomitant fear among healthcare providers. There are few, if any, recent studies which systematically examine this phenomenon in northern India, and any reports are largely limited to news media. Despite the fact that Bihar contributes the second highest number of new annual HIV infections in India (10\%), the bulk of studies dealing with stigma and discrimination focus on South India and Western India. ${ }^{10-17} 19$

Other studies examining the situation in India support our findings of non-consensual disclosure of HIV status to family members, and further report extreme precautionary measures such as burning bedding on discharge, billing the patient for the cost of infection control measures, and interacting with patients using gloves regardless of level of physical contact. ${ }^{20}{ }^{21}$ This is consistent with our study findings as well, which detail the poor adoption of universal precautions and patient perceptions of stigma associated with extra precautionary measures in selective cases. The literature suggests that such experiences can be very detrimental to treatment progress. A systematic review and meta-synthesis of 75 studies suggests a strong link between HIV-related stigma and compromised ART adherence ${ }^{22}$ Previous studies have documented the desires of patients to conceal their HIV status in contexts where HIV infection is heavily stigmatised. ${ }^{23-25}$ This has subsequently been linked to patients' unwillingness to access healthcare and poor mental health outcomes in different countries across the world. ${ }^{26-29}$ Fear of stigma and rejection from society further compounds the problem of refusal and referrals of PLHA in the healthcare system. ${ }^{30} 31$

Based on our findings, we first consider that there is a pressing need to improve the knowledge of HIV transmission, and in parallel, ensure implementation of universal precautions across all health facilities in the state, not just to reduce stigma and discrimination, but also to ensure proper infection control. Testing patients routinely for HIV as a precondition to accessing care is both stigmatising and unreliable as a measure, given that patients may be in the window period during the clinical presentation and other blood transmitted diseases could be missed by this measure. Current practices contribute to frequent referrals of patients, irrespective of medical need, and a pervasive fear of occupational exposure to HIV.

Second, we argue that the health system needs to take a stronger stance in both recognising and addressing stigma at the health facility level. However, before legal measures can be used, gaps in knowledge and practices among the physicians and other healthcare providers need to be addressed; simply put, staff need to be educated before punitive measures can be either appropriate or effective. The health system must take an active role in increasing the level of knowledge and support for healthcare providers, and implementing auditing measures to monitor ongoing practices. It must also increase networking efforts with PLHIV groups and other organisations working to improve access to care for key populations across Bihar in an effort to raise awareness about the new law and procedures to avail protection. Legal measures must go hand in hand with capacity building both in terms of knowledge and awareness, as well as resource provision.

Third, numerous evidence-based stigma reduction strategies and measures are mentioned in the global and Indian literature around HIV/AIDS which can be tried and tested in Bihar. ${ }^{32-34}$ The International Center for Research on Women and the United Nations Development Programme have already pilot tested an existing global HIV stigma reduction framework in five different settings in India across Maharashtra, Gujarat and Karnataka. ${ }^{32}$ Such existing frameworks should be tested and subsequently implemented in Bihar.

\section{LIMITATIONS}

The results of our study are based on a triangulation of interviews and field observations across two districts in Bihar, but our study is not without limitations. The research team did not ask providers and patients about intersecting stigma in PLHA who also had other stigmatised identities, such as transgender individuals, MSM or injecting drug users. We were unable to interview these key populations as they were much harder to target and our study design only focused on patients and community members who presented to healthcare facilities, which may present a selection bias. Future studies in Bihar should include PLHA networks and key populations outside healthcare facilities.

\section{CONCLUSION}

The continued presence of discriminatory and stigmatising attitudes towards PLHA is deeply concerning, and negatively impacts both disclosure of HIV status as well as access to care and treatment. It is likely that this pervasive discrimination may result in poorer outcomes for these patients, and may be implicated in the relatively high HIV-related mortality rate in Bihar. In order to improve treatment adherence and encourage optimal utilisation of services, it is imperative that the health system invest more in stigma reduction in the state of Bihar.

Acknowledgements This study would not have been possible without the dedicated efforts of the entire MSF field team in Bihar. We are grateful to Pallavi Lohani, Anuradha Ratan, Apurv Agrawal and all medical interns and residents at the All India Institute of Medical Sciences (Patna) for their support in the study.

Contributors SB conceptualised the study. MN, SP and PK conducted the study, while MN and PK managed data collection and analysis. MN, SB, LM-P, AH and SK designed and reviewed data collection tools. SP and PK revised the literature 
review. MN drafted the manuscript. All authors reviewed, edited and approved the final manuscript.

Funding The authors have not declared a specific grant for this research from any funding agency in the public, commercial or not-for-profit sectors.

Competing interests None declared.

Patient consent for publication Not required.

Ethics approval Ethics approval was obtained by the Medecins Sans Frontieres Ethics Review Board and the Institutional Ethics Committee at All India Institute of Medical Sciences, Patna.

Provenance and peer review Not commissioned; externally peer reviewed.

Data availability statement Data are available on reasonable request.

Open access This is an open access article distributed in accordance with the Creative Commons Attribution Non Commercial (CC BY-NC 4.0) license, which permits others to distribute, remix, adapt, build upon this work non-commercially, and license their derivative works on different terms, provided the original work is properly cited, appropriate credit is given, any changes made indicated, and the use is non-commercial. See: http://creativecommons.org/licenses/by-nc/4.0/.

ORCID iD

Sakib Burza https://orcid.org/0000-0001-7886-8633

\section{REFERENCES}

1 The Joint United Nations Programme on HIV/AIDS. Fact sheet stigma and discrimination, 2018. Available: http://data.unaids.org/ publications/fact-sheets03/fs_stigma_discrimination_en.pdf

2 The Joint United Nations Programme on HIV/AIDS. The gap report, 2014. Available: http://www.unaids.org/sites/default/files/media_ asset/UNAIDS_Gap_report_en.pdf

3 National AIDS Control Organization. National integrated biological and behavioural surveillance (IBBS) 2014-15 high risk groups, 2015. Available: http://www.aidsdatahub.org/sites/default/files/highlightreference/document/India_IBBS_report_2014-15.pdf

4 Steward WT, Herek GM, Ramakrishna J, et al. Hiv-Related stigma: adapting a theoretical framework for use in India. Soc Sci Med 2008;67:1225-35.

5 Steward WT, Bharat S, Ramakrishna J, et al. Stigma is associated with delays in seeking care among HIV-infected people in India. $J$ Int Assoc Provid AIDS Care 2013;12:103-9.

6 Golub SA, Gamarel KE. The impact of anticipated HIV stigma on delays in HIV testing behaviors: findings from a community-based sample of men who have sex with men and transgender women in New York City. AIDS Patient Care STDS 2013;27:621-7.

7 Turan B, Budhwani H, Fazeli PL, et al. How does stigma affect people living with HIV? the mediating roles of internalized and anticipated HIV stigma in the effects of perceived community stigma on health and psychosocial outcomes. AIDS Behav 2017;21:283-91.

8 Earnshaw VA, Bogart LM, Laurenceau J-P, et al. Internalized HIV stigma, art initiation and HIV-1 RNA suppression in South Africa: exploring avoidant coping as a longitudinal mediator. J Int AIDS Soc 2018;21:e25198.

9 Rice WS, Crockett KB, Mugavero MJ, et al. Association between internalized HIV-related stigma and HIV care visit adherence. J Acquir Immune Defic Syndr 2017;76:482-7.

10 Charles B, Jeyaseelan L, Pandian AK, et al. Association between stigma, depression and quality of life of people living with HIV/AIDS (PLHA) in South India - a community based cross sectional study. BMC Public Health 2012;12:463.

11 Datta S, Bhattacherjee S, Sherpa PL, et al. Perceived HIV related stigma among patients attending art center of a tertiary care center in rural West Bengal, India. J Clin Diagn Res 2016;10:VC09-12.

12 Joglekar N, Paranjape R, Jain R, et al. Barriers to ART adherence \& follow ups among patients attending ART centres in Maharashtra, India. Indian J Med Res 2011;134:954.
13 Kumarasamy N, Safren SA, Raminani SR, et al. Barriers and facilitators to antiretroviral medication adherence among patients with HIV in Chennai, India: a qualitative study. AIDS Patient Care STDS 2005;19:526-37.

14 Sivaram S, Zelaya C, Srikrishnan AK, et al. Associations between social capital and HIV stigma in Chennai, India: considerations for prevention intervention design. AIDS Educ Prev 2009;21:233-50.

15 Ekstrand ML, Ramakrishna J, Bharat S, et al. Prevalence and drivers of HIV stigma among health providers in urban India: implications for interventions. J Int AIDS Soc 2013;16:18717.

16 Thomas BE, Rehman F, Suryanarayanan D, et al. How stigmatizing is stigma in the life of people living with HIV: a study on HIV positive individuals from Chennai, South India. AIDS Care 2005;17:795-801.

17 Latkin C, Srikrishnan AK, Yang C, et al. The relationship between drug use stigma and HIV injection risk behaviors among injection drug users in Chennai, India. Drug Alcohol Depend 2010;110:221-7.

18 Ministry of Law and Justice. TheHuman immunodeficiency virus and acquired immune deficiency syndrome (prevention and control) act, 2017, 2017. Available: http://naco.gov.in/sites/default/files/HIV\% 20AIDS\%20Act.pdf

19 HIV Estimations. Technical Report. National AIDS Control Organization \& ICMR-National Institute of Medical Statistics, 2017. Available: http://naco.gov.in/sites/default/files/HIV\%20Estimations\% 202017\%20Report_1.pdf

20 Mahendra VS, Gilborn L, Bharat S, et al. Understanding and measuring AIDS-related stigma in health care settings: a developing country perspective. Sahara J 2007;4:616-25.

21 Nyblade L, Stangl A, Weiss E, et al. Combating HIV stigma in health care settings: what works? J Int AIDS Soc 2009;12:15.

22 Katz IT, Ryu AE, Onuegbu AG, et al. Impact of HIV-related stigma on treatment adherence: systematic review and meta-synthesis. J Int AIDS Soc 2013;16:18640.

23 George MS, Lambert $\mathrm{H}$. 'I am doing fine only because I have not told anyone': the necessity of concealment in the lives of people living with HIV in India. Cult Health Sex 2015;17:933-46.

24 Pandya SK. Patients testing positive for HIV-ethical dilemmas in India. issues in medical ethics. April-June 1997.5 (2). P. 49-55.. Issues Med Ethics 1997;5:49-55.

25 Karim QA, Meyer-Weitz A, Mboyi L, et al. The influence of AIDS stigma and discrimination and social cohesion on HIV testing and willingness to disclose HIV in rural KwaZulu-Natal, South Africa. Glob Public Health 2008;3:351-65.

26 Dapaah JM, Senah KA. Hiv/Aids clients, privacy and confidentiality; the case of two health centres in the Ashanti region of Ghana. BMC Med Ethics 2016;17:41.

27 Olalekan A. Conflicts in rights of disclosure of HIV status in South Western Nigeria: the health care provider's perspective. Mater Sociomed 2012;24:21.

28 Jayarajan N, Chandra PS. Hiv and mental health: an overview of research from India. Indian J Psychiatry 2010;52:S269.

29 Gyamfi E, Okyere P, Enoch A, et al. Prevalence of, and barriers to the disclosure of HIV status to infected children and adolescents in a district of Ghana. BMC Int Health Hum Rights 2017;17:8.

30 Rintamaki LS, Davis TC, Skripkauskas S, et al. Social stigma concerns and HIV medication adherence. AIDS Patient Care STDS 2006;20:359-68.

31 Mahajan AP, Sayles JN, Patel VA, et al. Stigma in the HIV/AIDS epidemic: a review of the literature and recommendations for the way forward. AIDS 2008;22:S57-65.

32 United Nations Development Programme. A global HIV stigma reduction framework adapted and implemented in five settings in India- summary report, 2013. Available: http://www.in.undp.org/ content/dam/india/docs/HIV_and_development/a-global-hiv-stigmareduction-framework-summary-report.PDF

33 EngenderHealth. Reducing stigma and discrimination related to HIV and AIDS: training for health care workers, 2004. Available: https:// www.k4health.org/sites/default/files/Reducing\%20Stigma\%20and\% 20Discrimination\%20Related\%20to\%20HIV\%20AIDS\%20for\% 20health\%20care\%20workers\%20English.pdf

34 The Population Council Inc. The PLHA-Friendly achievement checklist, 2003. Available: https://www.popcouncil.org/uploads/pdfs/ horizons/pfechklst.pdf 